\title{
ON THE CONSTITUTION OF POLAR FIBRE-REINFORCED MATERIALS
}

\author{
K.P. Soldatos \\ School of Mathematical Sciences, University of Nottingham, Nottingham NG7 2RD, UK \\ M.H.B.M. Shariff \\ Department of Mathematics, Khalifa University of Science and Technology, Abu Dhabi, UAE \\ J. Merodio \\ Department of Continuum Mechanics and Structures, Escuela de Caminos, Canales y Puertos, \\ Universidad Politecnica de Madrid, 28040, Madrid, Spain
}

\begin{abstract}
This article presents important constitutive refinements and simplifications in the theory of polar elasticity of materials reinforced by a single family of fibres resistant in bending. One of these simplifications is achieved by paying attention to forms of the strain energy which are symmetric with respect to the symmetric and the antisymmetric parts of the fibre gradient tensor. This leads to the identification of a restricted version of the theory that is predominantly influenced by the fibresplay mode of deformation. The lack of ellipticity of the governing equations of polar elasticity and the anticipation of existence of weak discontinuity surfaces even in the small deformation regime are also investigated. The manner in which potential activation of such surfaces is related with the action of either the fibre-bending or the fibre-splay deformation mode, as well as with their conjoined combination and coupling with their fibre-twist counterpart, is examined. The proposed constitutive equations can be simplified via the use of a new set of fourteen independent spectral invariants of the deformation. This set serves as an irreducible functional basis of relevant invariants or as an irreducible integrity basis of polynomial invariants. For instance, its use here enables identification of fourteen classical invariants that emerge as mutually independent from the known set of thirty-three in total classical invariants. In the special case of polynomial invariants, this result paves the way for identification of a corresponding minimal integrity basis.
\end{abstract}

Keywords: Cosserat elasticity, Fibre-reinforced materials, Fibres resistant in bending, Polar hyperelasticity, Polar elasticity, Spectral invariants, Weak discontinuity surfaces.

\section{Introduction}

In their endeavour to improve the theory of elastic materials reinforced by perfectly flexible fibres (initiated by Adkins and Rivlin [1]), Spencer and Soldatos [2] made the constitutive assumption that the elastic strain energy depends not only on the deformation and the vector of fibre direction, but also on the spatial derivative of the deformed fibre vector, subject to appropriate invariance requirements; see also [3]. They thus extended the theory and its region of applicability by making it able to model the behaviour of elastic materials with embedded fibres that possess bending stiffness [2]. The extended theory requires the inclusion of couple-stress and non-symmetric stress and is accordingly classified as a Cosserat-type theory [4].

The spatial derivative of the deformed fibre vector is a non-symmetric fibre-deformation tensor and enters the strain energy function via its symmetric and anti-symmetric parts, together with 
the Cauchy-Green deformation tensor and the fibre vector. The standard use of the theory of anisotropic tensor representations (e.g., [5]) implies that, for large elastic deformations, the strain energy may depend on at most thirty-three "classical" invariants. For self-sufficiency of this investigation, these are listed in Appendix A. In what follows, these are referred to as "classical invariants" and are quoted from the Appendix of [2], where it is also anticipated that their set is complete but may include redundant elements.

Reference [2] presented also a linearised version of the theory, which is applicable in cases that both the strains and the relevant fibre deformation measures (termed for simplicity "curvaturestrains") are infinitesimally small. The polar theory of transversely isotropic linear elasticity introduces a new set of seven additional elastic moduli, which complement the standard set of five elastic moduli met in conventional (non-polar) transverse isotropic linear elasticity. Hence, the theory is able to capture, and to account for three different modes of fibre deformation response which are loosely termed as the fibre-bending, the fibre-splay and fibre-twist mode. It is recalled that precisely the same version of transversely isotropic polar linear elasticity was produced later independently [6] in an alternative manner, by employing as point of theoretical departure the natural consideration of a strain energy function that is quadradic in the small strains and the couple-strains.

For large elastic deformations, Reference [2] also introduced a restricted version of the nonlinear theory, where the strain energy is predominantly influenced by the fibre-bending deformation and, inevitably, undermines the effects due to fibre-splay and fibre-twist. This restriction is done by replacing the nonsymmetric fibre-gradient tensor with the curvature vector of the deformed fibres. It thus led to a considerable reduction of the number of both the invariants involved in the nonlinear theory (from thirty-three to eleven) and the additional elastic moduli that enter its linearised version (from seven to one). For reasons that will become more evident in what follows, this will be referred to as the fibre-bending version of the theory or its restricted version that is predominantly influenced by the fibre-bending deformation mode. Due to its relative simplicity, the fibre-bending mode version has naturally attracted more attention in boundary value problem applications [7-15] that followed the publication of [2].

The experience gained from this activity [7-15] enables the present study to turn back into both the linear and the nonlinear versions of the initial, unrestricted form of the theory and, through appropriate refinement, to look for further possible simplifications of its constitutive equations. This refinement is achieved by (i) considering that the symmetric and the antisymmetric parts of the fibre gradient tensor enters the elastic strain energy in a symmetrical manner; and (ii) showing that only fourteen of the aforementioned thirty-three invariants are independent and, hence, that there exist nineteen relationships among the thirty-three classical invariants.

The refinement that stems from the first of these considerations becomes easier to handle and understand in the case of the linear theory which is thus considered first, in Sections 3 and 4. Section 2 serves previously as a proper reminder of the progress made so far in the theory, it further clarifies the targets set in this investigation and, where appropriate, provides connections discovered in [14] with the generally anisotropic polar linear elasticity due to Mindlin and Tiersten [16].

Section 3 then produces a further restricted version of the linearised theory described in References [2, 6], which now excludes effects due to fibre-bending and fibre-twist, and, hence, considers that the strain energy is predominantly influenced by the fibre-splay deformation mode. This newly introduced version will accordingly be referred to as the restricted version that captures the fibre-splay deformation mode only or the fibre-splay version of the theory.

An important feature that is common in all three versions of the linearised theory, namely its unrestricted and both of its restricted fibre-bending and fibre-splay versions, is that the resulting governing differential equations of are not elliptic. This lack of ellipticity is never met in non-polar linear elasticity (e.g., $[17,18]$ ) and is discussed afterwards in Section 4. It is recalled that ellipticity of the governing equations guarantees that a well-posed boundary value problem has always a unique solution in non-polar linear elasticity. Section 4 thus relates the observed lack of ellipticity with the existence and identification of a finite number of material surfaces, termed weak discontinuity 
surfaces, on which the anticipated continuous displacement field may possess discontinuous gradients. It is recalled in this context [19-21], that existence of weak discontinuity surfaces in an elastic material may cause activation of potential additional solutions that represent certain types of fibre damage modes; see also [14].

The refinement stemming from the second of the afore-mentioned considerations refers to the non-linear version of the unrestricted theory and is considered in Section 5. It requires an appropriate extension, and application onto the present polar hyperelasticity case, of a spectral analysis method introduced and employed in relevant cases met in non-polar hyperelasticity (e.g. [22-25]). The analysis presented in Section 5 is mainly based on the most recent relevant developments described in $[24,25]$ and, due to specific challenges met in spectral analysis developments, makes no use of the standard summation convention of repeated indices employed previously in Sections 2-4. Section 6 summarises the main conclusions drawn from the present study and briefly outlines directions of future potential development in the general subject of this communication.

\section{Preliminary theoretical concepts}

In this paper, all vector and tensor components are Cartesian components with respect to a fixed basis. Consider that a material particle with initial position $\boldsymbol{X}$ and coordinates $X_{R}$ moves during deformation of its surrounding hyperelastic solid to the position $\boldsymbol{x}$ with co-ordinates $x_{i}$ (subscripts take values 1,2 and 3) and, in the usual manner, assume that the implied deformation is described by the rule

$$
x_{i}=x_{i}\left(X_{R}\right) \text {. }
$$

The material of the solid is assumed locally transverse isotropic, due to the presence of a single family of embedded fibres defined by their direction unit vector $\boldsymbol{A}(\boldsymbol{X})$ or $\boldsymbol{a}(\boldsymbol{x})$ in the reference or the deformed configuration, respectively. The fibres are convected with the material, so that

$$
F_{i R} A_{R}=\lambda a_{i}=b_{i}, \quad \lambda^{2}=F_{i S} F_{i R} A_{R} A_{S}=\mathbf{A}^{T} \mathbf{C A},
$$

where $b_{i}$ are the components of deformed fibre vector, $\boldsymbol{b}, \lambda$ is the stretch in the fibre direction and

$$
F_{i Q}=\frac{\partial x_{i}}{\partial X_{Q}}=x_{i, Q}, \quad C_{Q S}=F_{i Q} F_{i S}=x_{i, Q} x_{i, S},
$$

are the components of the standard deformation gradient, $\boldsymbol{F}$, and the right Cauchy-Green deformation tensor, $\boldsymbol{C}$, respectively, and the standard summation convention od repeated indices applies.

The gradients of the deformed fibre vector are accounted for with the introduction of two tensor quantities, $\boldsymbol{G}$ and $\boldsymbol{\Lambda}$, with components

$$
\begin{aligned}
& G_{i R}=\frac{\partial b_{i}}{\partial X_{R}}=\frac{\partial}{\partial X_{R}}\left(\frac{\partial x_{i}}{\partial X_{Q}} A_{Q}\right)=\frac{\partial b_{i}}{\partial x_{j}} \frac{\partial x_{j}}{\partial X_{R}}, \\
& \Lambda_{Q R}=F_{i Q} G_{i R}=\frac{\partial x_{i}}{\partial X_{Q}} \frac{\partial b_{i}}{\partial X_{R}} .
\end{aligned}
$$

The resulting non-polar hyperelasticity of fibre-reinforced solids [2] requires consideration of couplestress and non-symmetric stress, whose action is coupled due to their simultaneous involvement in the equilibrium equations

$$
\sigma_{j i, j}=0, m_{j i, j}+\varepsilon_{i j k} \sigma_{j k}=0 \text {, }
$$

which are described in terms of the components of the Cauchy stress tensor, $\sigma$, the associated couplestress tensor, $\boldsymbol{m}$, and the three-dimensional alternating tensor, $\boldsymbol{\varepsilon}$.

\subsection{Constitutive equations of the non-linear theory}


The constitutive equations formulation is detailed in [2] and requires a split of the resulting nonsymmetric Cauchy stress tensor into its symmetric and antisymmetric parts $\sigma_{s}$ and $\sigma_{a}$, respectively. That formulation process leads to a set of constitutive equations which, in terms of components of these tensors, are as follows:

$$
\begin{aligned}
& \sigma_{i j}=\sigma_{(i j)}+\sigma_{[i j]}, \quad \sigma_{[i j]}=\frac{1}{2} \varepsilon_{k j i} m_{\ell k, \ell}, \\
& \sigma_{(i j)}=\frac{\rho}{\rho_{0}}\left\{F_{i R} F_{j S}\left(\frac{\partial W}{\partial C_{R S}}+\frac{\partial W}{\partial C_{S R}}\right)+\left(G_{i R} F_{j S}+G_{j R} F_{i S}\right) \frac{\partial W}{\partial \Lambda_{S R}}\right\}, \\
& \bar{m}_{\ell k}=\frac{2}{3} \frac{\rho}{\rho_{0}} \varepsilon_{k m n} \frac{\partial W}{\partial \Lambda_{P R}} F_{n P}\left(F_{\ell R} b_{m}+F_{m R} b_{\ell}\right),
\end{aligned}
$$

where, $W(\boldsymbol{C}, \boldsymbol{\Lambda}, \boldsymbol{A})$ represents the strain energy density per unit volume, $\rho$ and $\rho_{0}$ denote mass density in the deformed and undeformed configuration, respectively, and $\overline{\boldsymbol{m}}$ is the deviatoric part of $\boldsymbol{m}$.

As $W$ is an isotropic invariant of its tensorial arguments, it is expressible in terms of thirtythree invariants of the tensors $\boldsymbol{C}, \boldsymbol{\Lambda}_{s}, \boldsymbol{\Lambda}_{a}$ and the vector $\boldsymbol{A}$, where the appearing symmetric and antisymmetric parts of $\boldsymbol{\Lambda}$ are

$\Lambda_{s}=\left(\Lambda+\Lambda^{T}\right) / 2, \Lambda_{a}=\left(\Lambda-\Lambda^{T}\right) / 2$.

As the relevant list of invariants is read from Tables (e.g., [5]), it will be termed as list of classical invariants in what follows. While this is provided in the Appendix of [2], it is also quoted in Appendix A of the present study for the sake of self-sufficiency.

Spencer and Soldatos [2] realised, and made it clear, that this list of thirty-three invariants leads to excessively complicated constitutive equations. They, therefore, proposed further simplifying assumptions, and these led to (i) the afore mentioned restricted theory that is predominantly influenced by the fibre-bending deformation mode ([2], Section 6), and (ii) the linearised version of the theory ([2], Section 9) which was later re-derived in an alternative, more direct manner [6].

Nevertheless, neither of [2] and [6] considered that $\boldsymbol{\Lambda}_{s}$ and $\boldsymbol{\Lambda}_{a}$ may enter $W$ in the following symmetric manner

$W(\boldsymbol{C}, \boldsymbol{\Lambda}, \boldsymbol{A})=W_{(1)}\left(\boldsymbol{C}, \boldsymbol{\Lambda}_{s}, \boldsymbol{\Lambda}_{a}, \boldsymbol{A}\right)=W_{(1)}\left(\boldsymbol{C}, \boldsymbol{\Lambda}_{a}, \boldsymbol{\Lambda}_{s}, \boldsymbol{A}\right)$,

which emerges through strict consideration of the relationship $\boldsymbol{\Lambda}=\boldsymbol{\Lambda}_{s}+\boldsymbol{\Lambda}_{a}$, and is met later in Section 3. Instead of imposing this restriction on $W$, Reference [2] employed the more general consideration $W(\boldsymbol{C}, \boldsymbol{\Lambda}, \boldsymbol{A})=W_{(2)}\left(\boldsymbol{C}, \boldsymbol{\Lambda}_{s}, \boldsymbol{\Lambda}_{a}, \boldsymbol{A}\right) \neq W_{(2)}\left(\boldsymbol{C}, \boldsymbol{\Lambda}_{a}, \boldsymbol{\Lambda}_{s}, \boldsymbol{A}\right)$,

which is underpinned by postulations of the theory of representations for tensor functions (e.g., [5]). In fact, the unrestricted version of the corresponding linear theory, developed in Section 9 of Reference [2] and, in an alternative manner, in Reference [6], have also been developed under the general considerations that underpin (2.9); see also (2.15) below.

\subsection{Brief account of the linear theory and its constitutive equations}

In some detail, the linear theory makes naturally use of the displacement vector, $\boldsymbol{u}$, and the small strain and rotation tensors, $\boldsymbol{e}$ and $\boldsymbol{\omega}$, with components

$u_{i}=x_{i}-X_{i}, \quad e_{i j}=\frac{1}{2}\left(u_{i, j}+u_{j, i}\right), \quad \omega_{i j}=\frac{1}{2}\left(u_{i, j}-u_{j, i}\right)$,

respectively. Moreover, during the applied linearization process [6], $\boldsymbol{G}$ is replaced by a tensor $\boldsymbol{\kappa}$, whose components,

$\kappa_{i j}=\left(u_{i, k} a_{k}\right)_{, j}=u_{i, j k} a_{k}+u_{i, k} a_{k, j}$,

have dimensions of (length) ${ }^{-1}$ and, for convenience, are accordingly referred to as "curvature-strains" of the fibres. It is recalled that, due to the linear form of the resulting infinitesimal strain theory, the 
initial, $\boldsymbol{X}$, and final, $\boldsymbol{x}$, co-ordinate frames are here naturally regarded identical, as also are regarded the initial and final fibre direction vectors $\boldsymbol{A}$ and $\boldsymbol{a}$.

In linear elasticity, the strain energy function $W$ is necessarily quadratic in the considered principal kinematic variables. In the present theory, these variables include naturally the strains (2.10b), which are also involved in the non-polar case, and the curvature-strains (2.11). Hence, by considering $W$ quadratic in those sets of kinematic variables, the process followed in [6] concludes that $W$ is decomposed into two independent parts as follows:

$W(\mathbf{e}, \mathbf{\kappa}, \mathbf{a})=W^{e}(\mathbf{e}, \mathbf{a})+W^{\kappa}\left(\boldsymbol{\kappa}_{s}, \mathbf{\kappa}_{a}, \mathbf{a}\right)$,

where the components of the symmetric, $\boldsymbol{\kappa}_{s}$, and anti-symmetric, $\boldsymbol{\kappa}_{\boldsymbol{a}}$, parts of $\boldsymbol{\kappa}$ are, respectively,

$$
\begin{aligned}
& \kappa_{(i j)}=\frac{1}{2}\left(\kappa_{i j}+\kappa_{j i}\right)=\frac{1}{2}\left[\left(u_{i, k} a_{k}\right)_{, j}+\left(u_{j, k} a_{k}\right)_{, i}\right], \\
& \kappa_{[i j]}=\frac{1}{2}\left(\kappa_{i j}-\kappa_{j i}\right)=\frac{1}{2}\left[\left(u_{i, k} a_{k}\right)_{, j}-\left(u_{j, k} a_{k}\right)_{, i}\right] .
\end{aligned}
$$

Moreover, both $W^{e}$ and $W^{\kappa}$ are required to be even in $\boldsymbol{a}$, while, necessarily, $W^{e}$ should be strictly quadratic in the components of $\boldsymbol{e}$, and $W^{\kappa}$ strictly quadratic in the components of $\boldsymbol{\kappa}_{\boldsymbol{s}}$ and $\boldsymbol{\kappa}_{\boldsymbol{a}}$.

These considerations lead to a form of $W^{e}$ which is identical to its counterpart met in nonpolar transverse isotropic linear elasticity, and thus provide the constitutive equation for $\sigma_{s}$ in identical form to its well-known non-polar linear elasticity counterpart. On the other hand, and in the very context that enables the afore mentioned distinction between (2.8) and (2.9), $\boldsymbol{\kappa}_{s}$ and $\boldsymbol{\kappa}_{a}$ may be considered as entering $W^{\kappa}$ either in the symmetric manner

$$
W^{\kappa}(\boldsymbol{\kappa}, \boldsymbol{a})=W_{(1)}\left(\boldsymbol{\kappa}_{s}, \boldsymbol{\kappa}_{a}, \boldsymbol{a}\right)=W_{(1)}\left(\boldsymbol{\kappa}_{a}, \boldsymbol{\kappa}_{s}, \boldsymbol{a}\right) \text {, }
$$

or in a completely unrestricted manner that considers more generally that

$$
W^{\kappa}(\boldsymbol{\kappa}, \boldsymbol{a})=W_{(2)}\left(\boldsymbol{\kappa}_{s}, \boldsymbol{\kappa}_{a}, \boldsymbol{a}\right) \neq W_{(2)}\left(\boldsymbol{\kappa}_{a}, \boldsymbol{\kappa}_{s}, \boldsymbol{a}\right),
$$

Reference [6] as well as Section 9 of [2] consider only the more general form (2.15) of $W^{\kappa}$ which, being necessarily strictly quadratic in the components of $\boldsymbol{\kappa}_{s}$ and $\boldsymbol{\kappa}_{\boldsymbol{a}}$, is as follows:

$$
\begin{aligned}
W_{(2)}^{\kappa}= & \beta_{1}\left(\kappa_{n n}\right)^{2}+\beta_{2} \kappa_{n n} a_{k} \kappa_{(k m)} a_{m}+\beta_{3} \kappa_{(k m)} \kappa_{(m k)}+\beta_{4} a_{k} \kappa_{(k m)} \kappa_{(m n)} a_{n}+ \\
& \beta_{5} \kappa_{[k m]} \kappa_{[m k]}+\beta_{6} a_{k} \kappa_{[k m]} \kappa_{[m n]} a_{n}+\beta_{7} a_{k} \kappa_{(k m)} \kappa_{[m n]} a_{n}+\hat{\beta}_{3}\left(a_{k} \kappa_{(k m)} a_{m}\right)^{2} .
\end{aligned}
$$

The appearing coefficients, $\beta_{1}$ to $\beta_{7}$, and $\hat{\beta}_{3}$ (denoted as $\beta_{8}$ in [6]) are regarded as elastic moduli whose values depend on the ability of fibres to resist bending, as well as twist and splay types of deformation. This form of $W^{\kappa}$ yields the couple-stress constitutive equation

$$
\begin{aligned}
\bar{m}_{\ell r}= & \frac{2}{3} \varepsilon_{r \ell s} a_{s}\left(2 \beta_{1} \kappa_{n n}+\beta_{2} \kappa_{k m} a_{k} a_{m}\right)+\frac{2}{3} \varepsilon_{r i s} a_{s}\left(2 \beta_{3} \kappa_{(i \ell)}+\beta_{4} \kappa_{(i n)} a_{n} a_{\ell}\right)- \\
& \frac{1}{3} \varepsilon_{r i s}\left\{4 \beta_{5}\left(a_{s} \kappa_{[i \ell]}+a_{\ell} \kappa_{[i s]}\right)-2 \beta_{6} a_{n} a_{\ell}\left(a_{i} \kappa_{[s n]}-2 a_{s} \kappa_{[i n]}\right)+\beta_{7} a_{n} a_{\ell}\left(a_{i} \kappa_{n s}-2 a_{s} \kappa_{i n}\right)\right\},
\end{aligned}
$$

which, as is also justified in [6], is not influenced by the coefficient $\hat{\beta}_{3} \equiv \beta_{8}$.

Introduction of (2.17) and its standard $\sigma_{s}$-counterpart into the equilibrium equations (2.5) will then lead to the corresponding set of Navier-type displacement equations, which consist of a set of three linear partial differential equations (PDEs) for the three unknown components of the displacement vector, $\boldsymbol{u}$. In its most general form, that set of PDEs is described as follows:

$$
\hat{\mathcal{A}}_{\text {mij }} u_{j, m}+\mathcal{A}_{\text {mirj }} u_{j, m r}+C_{\text {mirjq }} u_{j, m r q}+\mathcal{B}_{\text {mirjinq }} u_{j, m r q n}=0 \text {, }
$$

where, $\hat{\mathcal{A}}_{\text {mij }}, \mathcal{A}_{\text {mirj }}, C_{\text {mirjq }}$ and $\mathcal{B}_{\text {mirinq }}$ are components of appropriate tensor quantities whose order is indicated by the number of associated indices. Explicit forms and values of the implied 
components depend on the shape of the fibres, namely on the form of $\boldsymbol{a}(\boldsymbol{x})$, as well as on whether the elastic moduli appearing in the constitutive equations are constant or variable in $\boldsymbol{x}$.

In principle, the form (2.18) of Navier-type displacement equations is reachable not only by the linear theory developed in [6], but also by any Cosserat-type linear elasticity theory. This observation thus includes the restricted, fibre-bending version of the present linear theory [2, 6, 8-15] and, of course, the generally anisotropic theory of polar linear elasticity due to Mindlin and Tiersten $[16]$.

It is emphasised for later use (see Section 4) that, unlike the present theoretical framework, Reference [16] does not consider cases of polar material anisotropy due to fibre presence. Instead, it refers to a state of general material anisotropy which, apart from the case of material isotropy, does not require consideration of any other type of material symmetry. Rather than using fibre-curvature gradients of the type (2.11), Reference [16] thus employs the spin vector, $\mathbf{\Omega}$, with components

$\Omega_{i}=\frac{1}{2} \varepsilon_{i j k} \omega_{k j}, \quad \omega_{k j}=\varepsilon_{i j k} \Omega_{i}$,

and uses its gradients to form an appropriate set of kinematic variables that enter the polar material counterpart of the strain energy function (2.12); see also [14].

\subsection{Straight fibres}

The particular case of straight fibres aligned parallel to the $x_{1}$-axis enabled Reference [6] to rearrange (2.16) in an alternative form which, under the assumption that $W^{\kappa}$, in general, and $W_{(2)}^{\kappa}$, in particular, is positive semi-definite, yields a set of inequalities that should necessarily hold by the values of the moduli $\beta_{1}$ to $\beta_{7}$ and $\hat{\beta}_{3}$. For self-sufficiency of the present study, as well as for later use, that alternative form of $W_{(2)}^{\kappa}$ is quoted in Appendix B along with the implied inequalities; see (B.1) and (B.3), respectively. Appendix B proceeds further and, also for later use, quotes from [14] the reason that makes the present unrestricted version of the theory incompatible with the theoretical framework of the Mindlin and Tiersten model [16].

As is also shown in Reference [6], the constitutive equation (2.17) simplifies in this case, in the sense that only the following couple-stress components are non-zero and they are:

$$
\begin{aligned}
& {\left[\begin{array}{c}
\bar{m}_{11} \\
\bar{m}_{22} \\
\bar{m}_{33}
\end{array}\right]=\left[\begin{array}{cc}
0 & 2 b_{3} \\
-b_{2} & b_{3} \\
b_{2} & b_{3}
\end{array}\right]\left[\begin{array}{l}
e_{23,1} \\
\Omega_{1,1}
\end{array}\right],} \\
& {\left[\begin{array}{c}
-\bar{m}_{32} \\
\bar{m}_{23} \\
-\bar{m}_{12} \\
\bar{m}_{13}
\end{array}\right]=\left[\begin{array}{ccccccc}
d_{11} & d_{22} & d_{33} & 0 & 0 & 0 & 0 \\
d_{11} & d_{33} & d_{22} & 0 & 0 & 0 & 0 \\
0 & 0 & 0 & d_{23} & d_{32} & 0 & 0 \\
0 & 0 & 0 & 0 & 0 & d_{23} & -d_{32}
\end{array}\right]\left[\begin{array}{l}
e_{11,1} \\
e_{22,1} \\
e_{33,1} \\
e_{31,1} \\
\Omega_{2,1} \\
e_{12,1} \\
\Omega_{3,1}
\end{array}\right],}
\end{aligned}
$$

where, the appearing elastic moduli relate as follows to their counterparts involved in (2.16): 


$$
\begin{aligned}
& b_{2}=\frac{4}{3} \beta_{3}, b_{3}=\frac{4}{3} \beta_{5}, d_{11}=\frac{2}{3}\left(2 \beta_{1}+\beta_{2}\right), d_{22}=\frac{4}{3}\left(\beta_{1}+\beta_{3}\right), d_{33}=\frac{4}{3} \beta_{1}, \\
& d_{23}=\frac{1}{3}\left(4 \beta_{3}+2 \beta_{4}+3 \beta_{7}\right), d_{32}=\frac{1}{3}\left(12 \beta_{5}+6 \beta_{6}-2 \beta_{4}-\beta_{7}\right) .
\end{aligned}
$$

By noting that the components of the spin vector and the infinitesimal rotation tensor are related in accordance with (2.19), it is not difficult for someone to verify that (2.20) is in complete agreement with its counterpart obtained in [6] as well as in Section 9 of [2]. The form of (2.20) thus shows that the seven couple stress components split into three groups, each one of which interacts independently with a corresponding set of kinematic variables. The couple-stresses that appear in (2.20a) correspond loosely to the so-called twist-mode met in the mechanics of liquid crystals, while the first and the second pair of couple-stresses in (2.20b) to the corresponding splay- and bendingmode, respectively. Nevertheless, a replacement of rotation gradients with their spin vector counterparts is imposed in (2.20), and this facilitates in what follows (see also Section 4 and Appendix B) the description of potential relations observed recently in [14] between the different versions of the present linear theory with the generally anisotropic polar linear elasticity due to Mindlin and Tiersten [16].

\section{Specialisation of the unrestricted linear theory: The fibre-splay deformation mode}

In line with the targets set in the Introduction, this Section aims to simplify the equations of the unrestricted version of the present linear theory by looking at the special case in which the symmetric and the antisymmetric parts of the fibre gradient tensor enter the elastic strain energy in the symmetrical manner implied by (2.14). It will thus be shown that this specialisation leads to a second type of a restricted linear theory, the polar elasticity part of which is influenced by the splay deformation mode of the fibres only.

\subsection{Fibres of general shape}

As is already mentioned, (2.16) is the most general form of $W^{\kappa}$ that is strictly quadratic in the symmetric and antisymmetric parts of $\boldsymbol{\kappa}$. In fact, (2.16) is an alternative form of the following expression:

$W_{(2)}^{\kappa}=\beta_{1} J_{1}^{2}+\beta_{2} J_{1} J_{2}+\hat{\beta}_{3} J_{2}^{2}+\beta_{3} J_{3}+\beta_{4} J_{4}+\beta_{5} J_{5}+\beta_{6} J_{6}+\beta_{7} J_{7}$,

where

$J_{1}=\operatorname{tr} \boldsymbol{\kappa}_{s}=\operatorname{tr} \boldsymbol{\kappa}, J_{2}=\mathbf{a} \boldsymbol{\kappa}_{s} \mathbf{a}=\mathbf{a} \boldsymbol{\kappa} \mathbf{a}, J_{3}=\operatorname{tr} \boldsymbol{\kappa}_{s}^{2}, J_{4}=\mathbf{a} \boldsymbol{\kappa}_{s}^{2} \mathbf{a}, J_{5}=\operatorname{tr} \boldsymbol{\kappa}_{a}^{2}, J_{6}=\mathbf{a} \boldsymbol{\kappa}_{a}^{2} \mathbf{a}, J_{7}=\mathbf{a} \boldsymbol{\kappa}_{s} \boldsymbol{\kappa}_{a} \mathbf{a}$,

is the subset of invariants that is formed by collecting from the corresponding complete invariant set all those elements which are at most quadratic in the curvature-strains [6].

It can readily be verified that upon imposing in the appearing coefficients the relations

$\beta_{3}=\beta_{5}, \quad \beta_{4}=\beta_{6}=\beta_{7} / 2$,

and taking also into consideration that

$\kappa_{(\mathrm{km})} \kappa_{[\mathrm{mk}]}=a_{k} \kappa_{[\mathrm{km}]} a_{m}=0$,

(2.16) reduces to the following quadratic expression for $\boldsymbol{\kappa}$ :

$W_{(1)}^{\kappa}=\beta_{1}\left(\kappa_{n n}\right)^{2}+\beta_{2} \kappa_{n n} a_{k} \kappa_{k m} a_{m}+\beta_{3} \kappa_{k m} \kappa_{m k}+\beta_{4} a_{k} \kappa_{k m} \kappa_{m k} a_{n}+\hat{\beta}_{3}\left(a_{k} \kappa_{k m} a_{m}\right)^{2}$,

which thus is shown a special case of (3.1) and, therefore, of (2.16).

However, a combination of (3.3) with the holding inequalities (B.3c-g) imposes on (3.4) the restrictions 
$\beta_{3} \geq 0, \quad \beta_{4} \geq 0, \quad \beta_{3} \leq 0, \quad \beta_{4} \leq-2 \beta_{3}, \quad \beta_{7}^{2}+4\left(2 \beta_{3}+\beta_{4}\right)^{2} \leq 0$,

which can hold only if

$\beta_{3}=\beta_{4}=\beta_{7}=0$.

The form (3.4) is then reduced to

$$
\begin{aligned}
W_{(1)}^{\kappa}=\beta_{1} J_{1}^{2}+\beta_{2} J_{1} J_{2}+\hat{\beta}_{3} J_{2}^{2} & =\beta_{1}\left(\kappa_{n n}\right)^{2}+\beta_{2} \kappa_{n n} a_{k} \kappa_{k m} a_{m}+\hat{\beta}_{3}\left(a_{k} \kappa_{k m} a_{m}\right)^{2} \\
& =\beta_{1}\left(\kappa_{(n n)}\right)^{2}+\beta_{2} \kappa_{(n n)} a_{k} \kappa_{(k m)} a_{m}+\hat{\beta}_{3}\left(a_{k} \kappa_{(k m)} a_{m}\right)^{2},
\end{aligned}
$$

and, consequently, the constitutive equation (2.17) simplifies and becomes

$\bar{m}_{\ell r}=\frac{2}{3} \varepsilon_{r \ell s} a_{s}\left(2 \beta_{1} \kappa_{n n}+\beta_{2} \kappa_{k m} a_{k} a_{m}\right)=\frac{2}{3} \varepsilon_{r \ell s} a_{s}\left(2 \beta_{1} \kappa_{(n n)}+\beta_{2} \kappa_{(k m)} a_{k} a_{m}\right)$.

Clearly, the antisymmetric part of the curvature-strain tensor does not contribute into either the curvature part (3.7) of the strain energy function or the couple-stress constitutive equation (3.8). Rather than seven [2,6], only three independent elastic moduli are thus involved in the specialised curvature part (3.7) of $W$ and, by combining (3.3) and (3.6) with the inequalities (B.3), one finds that positive definiteness of the strain energy function is guaranteed if

$\beta_{1} \geq 0, \quad \hat{\beta}_{3} \geq \beta_{2}^{2} / 4 \beta_{1}$.

Nevertheless, only two of these moduli, namely $\beta_{1}$ and $\beta_{2}$, are involved actively in the resulting couple-stress constitutive equation (3.8). Evidently, this special case of the unrestricted theory disregards contributions emerging through action of the infinitesimal rotation tensor (2.19b) or, equivalent, the spin vector (2.19a).

\subsection{Straight fibres}

As a result of the outlined reduction and simplifications, (2.21) yield

$b_{2}=b_{3}=0, d_{11}=\frac{2}{3}\left(2 \beta_{1}+\beta_{2}\right), d_{22}=d_{33}=\frac{4}{3} \beta_{1}, \quad d_{23}=d_{32}=0$,

and the constitutive equations (2.19) thus reveal that, in the case of straight fibres aligned parallel to the $x_{1}$-axis, the only non-zero couple-stresses are

$\bar{m}_{23}=-\bar{m}_{32}=d_{11} e_{11,1}+d_{22}\left(e_{22,1}+e_{33,1}\right)$.

This result reveals further that, like its restricted, fibre-bending mode version (e.g. $[2,6]$ and Section 4.1 below), the present version represents a second type of a restricted theory that makes use of a different a pair of non-zero couple-stress components. However, unlike its fibre-bending mode counterpart, which employs a single active elastic modulus, the present version employs two active elastic moduli and relates the involved non-zero pair of couple-stresses (3.11) with the fibre-splay deformation mode.

More importantly, and unlike its fibre-bending mode counterpart $[2,6]$, the present fibresplay mode version makes no use of spin gradients and, hence, does not have direct connection with the anisotropic version of the polar linear elasticity due to Mindlin and Tiersten [16]; see also [14]. This observation becomes clearer by further noting that, in this case, the expression (B.1) employed in [6] attains the special form

$$
W_{(1)}^{\kappa}=\left(e_{11,1}, e_{22,1}, e_{33,1}\right)\left[\begin{array}{ccc}
D_{11} & D_{12} & D_{12} \\
D_{12} & D_{22} & D_{22} \\
D_{12} & D_{22} & D_{22}
\end{array}\right]\left(\begin{array}{l}
e_{11,1} \\
e_{22,1} \\
e_{33,1}
\end{array}\right) \geq 0,
$$

in which the spin gradients are also absent, and 
$D_{11}=\beta_{1}+\beta_{2}+\hat{\beta}_{3}, \quad D_{22}=D_{23}=\beta_{1}, \quad D_{12}=\beta_{1}+\beta_{2} / 2$

\section{Lack of ellipticity of the polar linear elasticity equations: Weak discontinuity surfaces}

In summary, there have now become available three versions of polar linear elasticity for fibrous composites that exhibit transverse isotropy due to an embedded family of fibres resistant in bending. These are:

(i) the completely unrestricted version $[2,6]$, which considers that the symmetric and the antisymmetric parts of the curvature-strain tensor enter the strain energy function in the independent manner implied by (2.15) and, hence, captures and couples effects due to all three, fibre-bending, fibre-splay and fibre-twist deformation modes;

(ii) a special/restricted case of the same (Section 3), which considers that the symmetric and the antisymmetric parts of the curvature-strain tensor enter the strain energy in a manner that satisfies the symmetry restriction implied by (2.14) and, as a result, accounts for the fibre-splay deformation mode but neglects effects due to fibre-bending and fibre-twist; and

(iii) the restricted version presented in [2] and employed afterwards in several applications [715], which neglects effects due to fibre-splay and fibre-twist and, hence, considers that the predominant and more influential fibre deformation mode is that of fibre-bending.

Despite their already outlined differences, all three polar elasticity versions share a common general form of Navier-type displacement equations, namely the form of expression (2.18).

It is now recalled that, in the case of perfectly flexible of fibres, where $C_{\text {miriq }}=\mathcal{B}_{\text {miring }}=0$, all three of the implied, different versions of (2.18) reduce to, and meet their common background set of elliptic PDEs met in transversely isotropic non-polar linear elasticity. The ellipticity condition that prevails in that case (e.g., [21]) guarantees that in non-polar linear elasticity any well-posed boundary value problem has a unique solution. It also guarantees that such a unique solution is described in terms of continuous displacements whose derivatives of all orders are also continuous.

However, the set of equations (2.18) is not elliptic in the present, polar elasticity case. This is due to the appearance of fourth-order derivatives and, in particular, to the fact that, regardless of the employed version of the theory, not all of the non-zero components $B_{\text {mirnn }}$ are of the same sign. As is detailed elsewhere (e.g., $[6,14,15,19-21]$ ), the noticed lack of ellipticity can lead to identification of internal material surfaces, known as weak-discontinuity surfaces, on which displacements are still continuous but may possess discontinuous derivatives. In this context, the remaining of this Section aims to outline the current stage of knowledge regarding the manner that lack of ellipticity of (2.18) affects each of the afore mentioned versions of the theory.

It is accordingly recalled [6] that it is sufficient for the present purposes to temporarily consider that fibres are straight and, also, that all fibre resistance moduli are constant. This is because, if the fibres are curved or their resistance moduli vary with position, additional terms generated through the differentiations shown in (2.5), (2.6b), (2.11) and (2.13) do not affect $B_{\text {mirnn }}$ but, instead, are assembled within the remaining terms that appear in the left-hand-side of (2.18).

It is finally briefly recalled $[5,16,17]$ that identification of a weak discontinuity surface requires determination of its unit normal, $\boldsymbol{n}$. Accordingly, denote the jump of $u_{j, m r q n}$ across a surface with

$\left[\left[u_{j, m r q n}\right]\right]=k_{j} n_{m} n_{r} n_{q} n_{n}$

where $n_{n}$ are the components of $\boldsymbol{n}$ and $\boldsymbol{k}$, with components $k_{j}$, represents the amplitude of that jump. The difference of (2.18) on the two sides of the surface leads to a generalised eigenvalue problem of the form 
$\mathcal{B}_{\text {mirjqn }} k_{j} n_{m} n_{r} n_{q} n_{n}=0$ or $P_{i j}(\boldsymbol{n}) k_{j}=0$.

If at all present, the unit vector $\boldsymbol{n}$ is then required to emerge as a real solution of the characteristic algebraic equation

$\operatorname{det} \boldsymbol{P}(\boldsymbol{n})=0$.

\subsection{Version (iii) of the theory: Fibre-bending deformation mode}

The fibre-bending mode version is the simplest version of the present linear theory, as it makes active use of a single fibre stiffness modulus only. Moreover, this is the only version of the theory which is consistent with the generally anisotropic polar elasticity formalism due to Mindlin and Tiersten [16]. This is because imposition of certain conditions that suit transverse isotropy (see [14], Section 5) enables reduction of the spin-gradient part of $W$ employed in [16] into its curvature-strain counterpart,

$W^{\kappa}=\frac{3}{8} d^{f} K_{j} K_{j}+\bar{\gamma}\left(a_{j} K_{j}\right)^{2}, \quad K_{i}=u_{i, k j} a_{k} a_{j}$,

of the present restricted version.

Here, $d^{f}>0$ stands for the single fibre-bending stiffness that enters actively the corresponding couple-stress tensor

$\bar{m}_{\ell r}=d^{f} \varepsilon_{r s i} K_{i} a_{\ell} a_{s}$.

As the purpose of the present analysis is content with consideration of straight fibres only, alignment of the $x_{1}$-axis with the fibre direction reveals that, in that case, (4.5a) yields only a pair of non-zero components, namely

$\bar{m}_{12}=-d^{f} K_{3}=-d^{f} u_{3,11}, \quad \bar{m}_{13}=d^{f} K_{2}=d^{f} u_{2,11}$.

Evidently, the additional elastic modulus appearing in (4.4), namely $\bar{\gamma}>0$, remains inactive in the sense that it does not enter (4.5) or the corresponding Navier-type PDEs.

As a result of these observations, all theorems and theoretical results obtained in Section 3 of [14] in association with the generally anisotropic polar linear elasticity of Mindlin and Tiersten [16] apply also in the present case of the fibre-bending mode version of the theory. It follows that there exists only a single solution of the restricted theory PDEs that is expressible in terms of continuous displacements that possess continuous derivatives of all orders (termed, for simplicity, as continuous solution in [14]).

On the other hand, identification of potential weak discontinuity surfaces requires initially completion of the calculations implied in the last term of the left-hand-side of (2.18). These calculations commence as follows:

$\mathcal{B}_{\text {mirjnq }} u_{j, m r q n}=\frac{1}{2} \varepsilon_{k j i} \bar{m}_{m k, m i}$,

and, with use of (4.5a) for constant $d^{d}$, lead to

$\mathcal{B}_{\text {mirjnq }} u_{j, \text { mrqn }}=\frac{1}{2} d^{f}\left(a_{j} u_{m}-a_{m} u_{j}\right)_{\text {,mnrt }} a_{n} a_{r} a_{t}$.

Upon assuming next that, (i) the displacement, $\boldsymbol{u}$, as well as its up to third-order derivatives are all differentiable functions, but (ii) $\boldsymbol{u}$ may possess discontinuous derivatives of the fourth-order on a potential weak discontinuity surface having unit normal $\boldsymbol{n}$, on finds that the generalised eigenvalue problem (4.2) attains the form

$\left(a_{j} k_{m}-a_{m} k_{j}\right) n_{m} n_{n} n_{r} n_{t} a_{n} a_{r} a_{t}=0$.

This evidently admits the triple solution 
$n_{r} a_{r}=0$,

and the additional solution

$\left(a_{j} k_{m}-a_{m} k_{j}\right) n_{m}=0$.

However, a combination of (4.9) and (4.10) reveals that the former is also a solution of the latter. This is because, by inserting (4.9) into (4.10), the latter equation reduces to $k_{m} n_{m}=0$,

thus showing that (i) every surface that contains the fibres is a weak discontinuity surface and, if activated during deformation, (ii) the relevant micro-damage mode that corresponds to the anticipated $u_{j, m r q n}$-jump takes place on that same surface.

The easiest way for one to see an alternative solution of (4.10) is by aligning the $x_{1}$-axis with the fibre direction. As $\boldsymbol{a}=(1,0,0)^{\mathrm{T}}$ in that case, (4.10) reduces to $k_{m} n_{m}=k_{1} n_{1}, \quad k_{2} n_{1}=k_{3} n_{1}=0$,

which can hold non-trivially for non-zero $\boldsymbol{k}$ only if $n_{1}=0$ or $k_{2}=k_{3}=0$. However, the former case, where $n_{1}=0$, satisfies (4.9) and is therefore already discussed. With $k_{2}=k_{3}=0$ and both $k_{1}$ and $n$ being arbitrary, the second case reveals that $\boldsymbol{a}$ and $\boldsymbol{k}$ are co-axial vectors and, hence, signifies a mode of potential micro-damage that takes place along the fibres.

\subsection{Version (ii) of the theory: Fibre-splay deformation mode}

As is detailed in Section 3, the fibre-splay version of the present model makes active use of two fibre stiffness moduli only. Its constitutive relation is accordingly felt marginally more complicated than its just discussed fibre-bending counterpart. However, a substantial theoretical difference stems from the evident incompatibility of the present version with the Mindlin and Tiersten model [16]. The replacement of the spin-gradient kinematic variables employed in [16] with the strain-gradients that emerged in (3.11) and (3.12) implies that none of the relevant theorems proved and the associated results discussed in [14] are directly applicable in the present fibre-splay polar elasticity model.

However, identification of potential weak discontinuity surfaces is still possible after (2.13) and (3.8) are inserted into (4.6). Upon completing the implied calculations for straight fibres and constant elastic moduli, this process yields

$$
\mathcal{B}_{\text {mirnin }} u_{j, m r q n}=\frac{1}{3} a_{r}\left\{2 \beta_{1}\left(a_{j} u_{m, i}-a_{i} u_{m, j}\right)_{, m r j}+\beta_{2}\left(a_{j} u_{m, i}-a_{i} u_{m, j}\right)_{, n r j} a_{m} a_{n}\right\},
$$

and finally leads to the generalized eigenvalue problem

$a_{r} n_{r}\left(n_{j} a_{j} n_{i}-a_{i}\right)\left(2 \beta_{1} n_{p}+\beta_{2} a_{m} n_{m} a_{p}\right) k_{p}=0$,

where $\boldsymbol{n}$ and $\boldsymbol{k}$ still signify the afore mentioned relevant vectors.

Evidently, (4.14) admits again the solution (4.9), which has been already discussed, along with

$n_{j} a_{j} n_{i}-a_{i}=0$

and

$\left(2 \beta_{1} n_{p}+\beta_{2} a_{m} n_{m} a_{p}\right) k_{p}=0$.

On the other hand, (4.15) suggests that the unit vectors $\boldsymbol{a}$ and $\boldsymbol{n}$ are coaxial and, therefore, necessarily identical. This case thus reveals that surfaces normal to the fibres are weak discontinuity surfaces but, unless accompanied with some additional condition, leaves the direction of the $u_{j, m r q n}$-jump unspecified and, therefore, arbitrary.

On writing (4.16) in a non-component form, one has

$2 \beta_{1} \boldsymbol{n} . \boldsymbol{k}+\beta_{2}(\boldsymbol{a} . \boldsymbol{n})(\boldsymbol{a} . \boldsymbol{k})=0$. 
It is thus seen that the first solution of (4.14), where $\boldsymbol{a} \cdot \boldsymbol{n}=\boldsymbol{n} \cdot \boldsymbol{k}=0$, is a special case of (4.17). Moreover, in the case that $\boldsymbol{a}$ and $\boldsymbol{n}$ are coaxial, (4.17) gives

$2 \beta_{1}+\beta_{2}=0$.

Evidently, this particular solution of (4.16) differs from its (4.15) counterpart, which is present regardless of the values of $\beta_{1}$ and $\beta_{2}$. What makes it though worth of notice is that, in the special case that (4.18) holds, (3.9) suggest that the strain energy function can be positive definite only if $\hat{\beta}_{3} \geq \beta_{1} \geq 0$.

4.3 Version (i) - Unrestricted theory: Full coupling of fibre-bending, -splay and -twist deformation modes

As is detailed in [14] and becomes obvious in either (2.20) or (B.4) - (B.6), the kinematic variables employed in the formulation of the unrestricted theory include gradients of both the strain tensor and the spin vector. A more detailed discussion of this difference between the unrestricted theory and its Mindlin and Tiersten counterpart [16] is presented in Section 6 of [14]. There, it is concluded that the evident incompatibility of the two theories require considerable and careful consideration, at least because, again, the relevant theorems proved in [14] are not directly applicable to the present unrestricted theoretical model.

However, Reference [6] has already detailed the manner that potential weak discontinuities are identified in cases that boundary value problems are modelled with use the unrestricted version of the theory. For self-sufficiency of the present communication, it is accordingly recalled that, in this case, the equation that corresponds to either (4.7) or (4.13) is as follows [6]:

$$
\begin{aligned}
\mathcal{B}_{\text {mirnm }} u_{j, \text { mrqn }}=a_{r}\left\{a_{i} \mid \hat{c}_{1} u_{n, n r j j}+a_{n} a_{m}\left(\hat{c}_{2} u_{n, r m j j}+\hat{c}_{3} u_{j, \text { rrmm }}\right)\right]+ \\
\left.a_{j}\left[\left(\hat{c}_{4} u_{i, r m m j}+\hat{c}_{5} u_{m, i r m j}\right)+a_{n} a_{m}\left(\hat{c}_{6} u_{i, r n m j}+\hat{c}_{7} u_{n, i r m j}\right)\right]\right\},
\end{aligned}
$$

where the appearing elastic moduli are as follows:

$$
\begin{aligned}
& \hat{c}_{1}=\frac{2}{3}\left(\beta_{1}+4 \beta_{3}\right), \quad \hat{c}_{2}=\frac{1}{3}\left(\beta_{2}+2 \beta_{4}+2 \beta_{6}\right), \quad \hat{c}_{3}=\frac{2}{3}\left(\beta_{4}-\beta_{6}+\beta_{7}\right), \\
& \hat{c}_{4}=\frac{4}{3} \beta_{5}, \quad \hat{c}_{5}=-\frac{2}{3}\left(\beta_{1}+2 \beta_{5}\right), \quad \hat{c}_{6}=\frac{1}{3} \beta_{6}, \quad \hat{c}_{7}=-\frac{1}{3}\left(\beta_{2}+\beta_{6}+\beta_{7}\right) .
\end{aligned}
$$

The corresponding generalised eigenvalue problem that enables identification of the vectors $\boldsymbol{n}$ and $\boldsymbol{k}$ is still of the form (4.3), where [6]

$$
\begin{aligned}
& P_{i j}(\boldsymbol{n})=a_{r} n_{r}\left\{\left[\hat{c}_{1} n_{j}+a_{m} n_{m}\left(\hat{c}_{2} a_{j}+\hat{c}_{3} a_{n} n_{n} n_{j}\right)\right] a_{i}+\right. \\
& \left.a_{s} n_{s}\left[\left(\hat{c}_{4}+\hat{c}_{6}\left(a_{q} n_{q}\right)^{2}\right) \delta_{i j}+\left(\hat{c}_{5} n_{j}+\hat{c}_{7} a_{m} n_{m} a_{j}\right) n_{i}\right]\right\} .
\end{aligned}
$$

Due to appearance of seven independent elastic moduli, potential solutions to the characteristic equation (4.3) should, in general, be sought numerically. However, by observing that $P_{i j}(\boldsymbol{n})$ is proportional to $a_{r} n_{r}$, it becomes understood that (4.3) admits again the solution (4.9) which revels that surfaces containing the fibres are surfaces of weak discontinuity.

It is finally noted that, by inserting (3.3) and (3.6) into (4.21), one can readily verify that

$\hat{c}_{1}=-\hat{c}_{5}=\frac{2}{3} \beta_{1}, \quad \hat{c}_{2}=-\hat{c}_{7}=\frac{1}{3} \beta_{2}, \quad \hat{c}_{3}=\hat{c}_{4}=\hat{c}_{6}=0$,

and, hence, in the particular case of the fibre-splay version of the theory, (4.22) is correctly reduced into (4.13). 


\section{Irreducible functional basis of invariants in the unrestricted version of the non-linear theory}

The second target set in the Introduction stems from a need for a simplification of the constitutive equation of the unrestricted version of the non-linear theory, which ideally requires identification of the redundant elements appearing in the complete set of the thirty-three classical invariants listed initially in [2] and, for convenience, quoted also in Appendix A. This Section serves as a first step towards this target by initially showing that, in general, only fourteen of these invariants are independent and, next, by identifying a subset of the fourteen classical invariants (A.1) that may be employed as a relevant irreducible functional basis. This result follows a proof of the fact that there exist nineteen relationships among the thirty-three classical invariants (A.1), but no attempt is presently made towards precise identification of those relationships.

The summation convention of repeated indices, which was employed systematically in previous Sections, is abandoned in this Section due to the complicated form of the employed equations. However, indices still take the values 1, 2 and 3, unless it is stated otherwise. Moreover, the indices "s" and "a" are excepted from this rule. Due to their strict association with $\Lambda$, these assume no numerical values but are instead reserved to denote the symmetric and antisymmetric part of that matrix, respectively.

\subsection{Spectral invariants}

As a starting point, consider the spectral description

$\boldsymbol{F}=\boldsymbol{R} \boldsymbol{U}=\sum_{i=1}^{3} \lambda_{i} \boldsymbol{R} \boldsymbol{u}_{i} \otimes \boldsymbol{u}_{i}, \quad \boldsymbol{C}=\sum_{i=1}^{3} \lambda_{i}^{2} \boldsymbol{u}_{i} \otimes \boldsymbol{u}_{i}$

of the deformation gradient and the Cauchy-Green deformation tensor, respectively, where the principal stretches, $\lambda_{i}$, and the vectors $\boldsymbol{u}_{i}$ are respectively the eigenvalues and the orthonormal eigenvectors of the symmetric matrix $\boldsymbol{U} ; \boldsymbol{R}$ is the corresponding antisymmetric matrix in the implied standard, unique polar decomposition of $\boldsymbol{F}$.

Using the vector basis $\boldsymbol{u}_{i}$, the form (2.9) of $W$, whose general dependency on $\boldsymbol{\Lambda}_{s}$ and $\boldsymbol{\Lambda}_{a}$ undermines the connection (2.7), can take the form

$W_{(2)}\left(\boldsymbol{C}, \boldsymbol{\Lambda}_{s}, \boldsymbol{\Lambda}_{a}, \boldsymbol{A}\right)=W_{(3)}\left(\lambda_{i}, s_{i j}, r_{i j}, A_{i}\right)$,

where, like $\lambda_{i}$ and $\boldsymbol{u}_{i}$, the quantities

$s_{i j}=s_{j i}=\boldsymbol{u}_{i}^{T} \boldsymbol{\Lambda}_{s} \boldsymbol{u}_{j}, \quad r_{i j}=-r_{j i}=\boldsymbol{u}_{i}^{T} \boldsymbol{\Lambda}_{a} \boldsymbol{u}_{j}, \quad r_{i i}=0, \quad A_{i}=\boldsymbol{u}_{i}^{T} \boldsymbol{A}$,

are all invariants under any orthogonal transformation, $\boldsymbol{Q}\left(\boldsymbol{Q}^{T} \boldsymbol{Q}=\boldsymbol{Q} \boldsymbol{Q}^{T}=\boldsymbol{I}\right)$. This is readily verified by noting that

$$
\begin{aligned}
& s_{i j}=\boldsymbol{u}_{i}^{T} \boldsymbol{\Lambda}_{s} \boldsymbol{u}_{j}=\boldsymbol{u}_{i}^{T}\left(\boldsymbol{Q}^{T} \boldsymbol{Q}\right) \boldsymbol{\Lambda}_{s}\left(\boldsymbol{Q}^{T} \boldsymbol{Q}\right) \boldsymbol{u}_{j}=\left(\boldsymbol{Q} \boldsymbol{u}_{i}\right)^{T}\left(\boldsymbol{Q} \boldsymbol{\Lambda}_{s} \boldsymbol{Q}^{T}\right)\left(\boldsymbol{Q u} \boldsymbol{u}_{j}\right), \\
& r_{i j}=\boldsymbol{u}_{i}^{T} \boldsymbol{\Lambda}_{a} \boldsymbol{u}_{j}=\boldsymbol{u}_{i}^{T}\left(\boldsymbol{Q}^{T} \boldsymbol{Q}\right) \boldsymbol{\Lambda}_{a}\left(\boldsymbol{Q}^{T} \boldsymbol{Q}\right) \boldsymbol{u}_{j}=\left(\boldsymbol{Q} \boldsymbol{u}_{i}\right)^{T}\left(\boldsymbol{Q} \boldsymbol{\Lambda}_{a} \boldsymbol{Q}^{T}\right)\left(\boldsymbol{Q u} \boldsymbol{u}_{j}\right), \\
& A_{i}=\boldsymbol{u}_{i}^{T} \boldsymbol{A}=\boldsymbol{u}_{i}^{T}\left(\boldsymbol{Q}^{T} \boldsymbol{Q}\right) \boldsymbol{A}=\left(Q \boldsymbol{u}_{i}\right)^{T}(\boldsymbol{Q A}) .
\end{aligned}
$$

As $s_{i j}$ has six, $r_{i j}$ three, and the unit vector $A_{i}$ only two independent components, $W_{(3)}$ is generally a function of the fourteen spectral invariants $\left\{\lambda_{i}, s_{i j}, r_{i j}, A_{\alpha}\right\}$, where the appearing Greek index implies that only two of the components of $A_{i}$ (say, $A_{1}$ and $A_{2}$ ) are independent. These invariants are independent except from a finite number of possible degenerated cases, such as those referred to below and in Appendix C. The spectral invariants $\left\{\lambda_{i}, s_{i j}, r_{i j}, A_{\alpha}\right\}$ can accordingly serve as 
an irreducible functional basis of invariants, in general, or as an irreducible integrity basis in the particular case of polynomial invariants.

\subsection{A set of fourteen independent classical invariants}

As $W_{(3)}$ is regarded a function of the fourteen spectral invariants, its equivalent form $W_{(2)}$ may be felt as function of the same number of independent invariants, thus leading to the conclusion that only fourteen of the thirty-three classical invariants (A.1) are functionally independent. Such a subset of fourteen independent classical invariants is revealed in what follows where, for the sake of clear distinction, its components will be renamed as $J_{1}, J_{2}, \ldots, J_{14}$.

One may thus begin with the well-known relations

$J_{1} \equiv I_{1}=\operatorname{tr} \boldsymbol{C}=\sum_{i=1}^{3} \lambda_{i}^{2}, \quad J_{2} \equiv I_{2}=\frac{1}{2}\left[(\operatorname{tr} \boldsymbol{C})^{2}-\operatorname{tr} \boldsymbol{C}^{2}\right]=\lambda_{1}^{2} \lambda_{2}^{2}+\lambda_{2}^{2} \lambda_{3}^{2}+\lambda_{3}^{2} \lambda_{1}^{2}$,

$J_{3} \equiv I_{3}=\operatorname{det} C=\lambda_{1}^{2} \lambda_{2}^{2} \lambda_{3}^{2}$,

which, upon inversion yield either

$$
\begin{aligned}
& \lambda_{j}=\frac{1}{\sqrt{3}}\left\{J_{1}+2 \sqrt{J_{1}^{2}-3 J_{2}} \cos \frac{1}{3}[\theta+2 \pi(j-1)]\right\}^{1 / 2}, \\
& \theta=\arccos \left[\frac{2 J_{1}^{3}-9 J_{1} J_{2}+27 J_{3}}{2\left(J_{1}^{2}-3 J_{2}\right)^{3 / 2}}\right], \quad J_{1}^{2}-3 J_{2} \neq 0,
\end{aligned}
$$

or

$$
\lambda_{j}=\frac{1}{\sqrt{3}}\left\{J_{1}+\left(27 J_{3}-J_{1}^{3}\right)^{1 / 3}\left[\cos \left(\frac{2}{3} \pi j\right)+\sqrt{-1} \sin \left(\frac{2}{3} \pi j\right)\right]\right\}^{1 / 2}, \quad J_{1}^{2}-3 J_{2} \neq 0 .
$$

The values thus obtained for the principal stretches, $\lambda_{i}$, may be either distinct or degenerated.

This Section considers only the former case and shows that only fourteen of the classical invariants listed in (A.1) are strictly independent. In particular cases that two or more of $\lambda_{i}$ are not distinct, it can be shown with no greater difficulty that the number of independent invariants is smaller than fourteen, as is exemplified in Appendix C.

\subsection{Distinct principal stretches}

It is initially noted that

$$
\begin{aligned}
& J_{4} \equiv I_{4}=\boldsymbol{A} \boldsymbol{C} \boldsymbol{A}=\lambda_{1}^{2} A_{1}^{2}+\lambda_{2}^{2} A_{2}^{2}+\lambda_{3}^{2} A_{3}^{2}, \\
& J_{5} \equiv I_{5}=\boldsymbol{A} \boldsymbol{C}^{2} \boldsymbol{A}=\lambda_{1}^{4} A_{1}^{2}+\lambda_{2}^{4} A_{2}^{2}+\lambda_{3}^{4} A_{3}^{2}, \\
& A_{1}^{2}+A_{2}^{2}+A_{3}^{2}=1 .
\end{aligned}
$$

Hence, by solving this algebraic system of linear equations for $A_{i}^{2}$, and making further use of (5.6), one will obtain

$$
A_{i}=A_{i}\left(J_{1}, J_{2}, J_{3}, J_{4}, J_{5}\right) \text {. }
$$

$$
\text { It is next seen that as }
$$




$$
\begin{aligned}
& J_{6} \equiv I_{6}=\operatorname{tr} \Lambda_{s}=\sum_{i=1}^{3} s_{i i}, \\
& J_{7} \equiv I_{10}=\operatorname{tr} C \Lambda_{s}=\sum_{i=1}^{3} \lambda_{i}^{2} s_{i i}, \\
& J_{8} \equiv I_{11}=\operatorname{tr} C^{2} \Lambda_{s}=\sum_{i=1}^{3} \lambda_{i}^{4} s_{i i},
\end{aligned}
$$

solution of this algebraic system of linear equations for $s_{i i}$ will similarly yield

$$
s_{i i}=s_{i i}\left(J_{1}, J_{2}, J_{3}, J_{6}, J_{7}, J_{8}\right) \text {. }
$$

It can be seen that

$$
\begin{aligned}
& J_{9} \equiv I_{7}=\operatorname{tr} \Lambda_{s}^{2}=s_{11}^{2}+s_{22}^{2}+s_{33}^{2}+2\left(s_{12}^{2}+s_{13}^{2}+s_{23}^{2}\right), \\
& J_{10} \equiv I_{12}=\operatorname{tr} C \Lambda_{s}^{2}=\lambda_{1}^{2}\left(s_{11}^{2}+s_{12}^{2}+s_{13}^{2}\right)+\lambda_{2}^{2}\left(s_{21}^{2}+s_{22}^{2}+s_{23}^{2}\right), \\
& J_{11} \equiv I_{13}=\operatorname{tr} C^{2} \Lambda_{s}^{2}=\lambda_{1}^{4}\left(s_{11}^{2}+s_{12}^{2}+s_{13}^{2}\right)+\lambda_{2}^{4}\left(s_{21}^{2}+s_{22}^{2}+s_{23}^{2}\right)+\lambda_{3}^{4}\left(s_{31}^{2}+s_{32}^{2}+s_{33}^{2}\right),
\end{aligned}
$$

and, by solving this algebraic system of linear equations for $s_{i j}^{2}=s_{j i}^{2}$, with $i \neq j$, one will obtain

$$
\begin{aligned}
& s_{i j}=s_{i j}\left(J_{1}, J_{2}, J_{3}, J_{6}, J_{7}, J_{8}, J_{9}, J_{10}, J_{11}\right)=s_{j i}, \quad(i \neq j) . \\
& \quad \text { Finally, as } \\
& J_{12} \equiv I_{8}=\operatorname{tr} \Lambda_{a}^{2}=2\left(r_{12}^{2}+r_{13}^{2}+r_{23}^{2}\right), \\
& J_{13} \equiv I_{14}=\operatorname{tr} C \Lambda_{a}^{2}=\lambda_{1}^{2}\left(r_{12}^{2}+r_{13}^{2}\right)+\lambda_{2}^{2}\left(r_{21}^{2}+r_{23}^{2}\right)+\lambda_{3}^{2}\left(r_{31}^{2}+r_{32}^{2}\right), \\
& J_{14} \equiv I_{15}=\operatorname{tr} C^{2} \Lambda_{a}^{2}=\lambda_{1}^{4}\left(r_{12}^{2}+r_{13}^{2}\right)+\lambda_{2}^{4}\left(r_{21}^{2}+r_{23}^{2}\right)+\lambda_{3}^{4}\left(r_{31}^{2}+r_{32}^{2}\right),
\end{aligned}
$$

solution of this algebraic system of linear equations for $r_{i j}^{2}=r_{j i}^{2}$, with $i \neq j$, will provide

$$
r_{i j}=r_{i j}\left(J_{1}, J_{2}, J_{3}, J_{12}, J_{13}, J_{14}\right)=-r_{j i}, \quad(i \neq j) \text {. }
$$

Each of the nineteen classical invariants remaining unused in the list (A.1) depends explicitly on the fourteen spectral invariants $\lambda_{i}, s_{i j}, r_{i j}$ and $A_{i}$. By virtue of (5.6), (5.8), (5.10), (5.12) and (5.14), each of those nineteen invariants is in principle expressible in terms of $J_{1}, J_{2}, \ldots, J_{14}$ and, by taking the appropriate signs they are single-valued functions of $J_{1}, J_{2}, \ldots, J_{14}$ and thus functionally reducible. Existence of nineteen such relationships among the thirty-three classical invariants then implies that, like its spectral counterpart, $\left\{\lambda_{i}, s_{i j}, r_{i j}, A_{\alpha}\right\}$, the functionally basis $\left\{J_{1}, J_{2}, \ldots, J_{14}\right\}$ is irreducible.

It is now noted that each of $J_{1}, J_{2}, \ldots, J_{14}$ is a polynomial expression of spectral invariants. It follows that in the rather unlikely case that each of the afore-mentioned nineteen single-valued invariant expressions is still polynomial in the remainder invariants, then the functional basis $\left\{J_{1}, J_{2}\right.$, $\left.\ldots, J_{14}\right\}$ is also an integrity basis which is both irreducible and minimal. However, if some, say $n$, of the implied nineteen relationships are syzygies $(0<n \leq 19)$, while the remaining $19-n$ relationships are polynomials, then $n$ of $I_{9}, I_{16}, I_{17}, \ldots, I_{33}$ are irreducible polynomial invariants. In that case, the minimal integrity basis contains $14+n$ invariants, and its first fourteen elements can still coincide with those of the irreducible functional basis $\left\{J_{1}, J_{2}, \ldots, J_{14}\right\}$. 


\section{Conclusions}

Constitutive modelling of large elastic deformations of elastic materials reinforced by a single family of unidirectional fibres resistant in bending requires use of thirty-three classical invariants of deformation [2]. As is shown in Section 6 though, the same constitutive equations may alternatively be described with use of fourteen independent spectral invariants. On its own right to serve as an irreducible functional basis of relevant invariants, that set of fourteen spectral invariants implies that there exist nineteen relationships among the thirty-three classical invariants introduced in [2]. Explicit forms of those relationships are not provided here, but a subset of fourteen independent classical invariants has indeed been identified. These new findings are expected to underpin further relevant progress and thus assist substantially the development and a better understanding of several important theoretical and practical issues involved in this non-linear polar theory of elasticity (hyperelasticity), as well as in its linearised (infinitesimal strain) counterpart.

Due its relative simplicity, as compared with its non-linear counterpart, the linear polar theory of elasticity has in fact naturally attracted more attention in recent publications [6-15]. Investigations regarding features as well as the region as its applicability have, for instance, already revealed some of its similarities and differences with the Mindlin and Tiersten theory [16]. Further relevant progress will thus feedback valuable information towards better understanding of relevant issues involved in the non-linear theory. It is recalled in this context [15] that the Mindlin and Tiersten formalism [16] is in remarkable agreement with the restricted, fibre-bending mode version of the linear theory. This agreement is based on the fact the polar (couple-stress) part of both formalisms employ the spin-gradients as kinematic variables.

However, the Mindlin and Tiersten framework [16] fails to agree with the unrestricted linear version of the present formalism which captures, and couples together effects due to all three fibrebending, fibre-splay and fibre-twist deformation modes. This is due to a set of additional, intrinsic kinematic variables which, although absent in the Mindlin and Tiersten theory, do appear along with their spin-gradient counterparts, and are made use of in the polar part of the present theory [15]. It has accordingly been revealing, in Section 3, to find out that this kind of intrinsic kinematic variables are employed on their own in the fibre-splay version of the present linear theory, namely in the complete absence of their spin-gradient counterparts. This revelation leads to the conclusion that, despite its generality, the degree of anisotropy involved in the Mindlin and Tiersten theory is unable to capture effects due to potential existence of fibre-splay deformation.

The existence of the afore mentioned different sets of kinematic variables observed in the linear theory, and their distinct association with corresponding, specific fibre deformation modes are not expected to be so evidently distinct when dealing with polar hyperelasticity problems. Their appearance and clear distinction in the region of applicability of infinitesimal strain theory may thus provide valuable feedback when dealing with finite strain problems, where non-linearity is expected to reinforce the coupling of, and to make thus indistinguishable the effects due to all three of the afore mentioned fibre deformation modes.

Another advantage of the relative simplicity of the linear theory is associated with the influence that the known lack of ellipticity of its governing equations may exert on the solution of relevant polar elasticity boundary value problems [19-21]. In this context, Section 4 detailed systematically the manner in which potential weak discontinuity surfaces are analytically identified in either of the restricted fibre-bending and fibre-splay versions of theory, and also in its unrestricted version where both fibre-bending and fibre-splay modes are coupled together with their fibre-twist counterpart. Still though, full comprehension of this subject requires considerable further and thorough investigation. In this regard, References $[14,15]$ have initiated a search towards collection of thoughts and new ideas regarding the manner in which potential weak discontinuity solutions may be sought and, if present in polar elasticity boundary value problems, be found in parallel, as well as in competition with their single fully continuous counterpart. 


\section{Appendix A: List of classical invariants employed in the unrestricted non-linear theory [2]}

The thirty-three invariants involved in the unrestricted version of the non-linear theory are also listed [2] and are as follows:

$I_{1}=\operatorname{tr} \mathbf{C}, \quad I_{2}=1 / 2\left\{(\operatorname{tr} \mathbf{C})^{2}-\operatorname{tr} \mathbf{C}^{2}\right\}, \quad I_{3}=\operatorname{det} \mathbf{C}, \quad I_{4}=\mathbf{A C A}, \quad I_{5}=\mathbf{A} \mathbf{C}^{2} \mathbf{A}$,

$I_{6}=\operatorname{tr} \boldsymbol{\Lambda}^{s}=\operatorname{tr} \boldsymbol{\Lambda}, I_{7}=\operatorname{tr} \boldsymbol{\Lambda}_{s}{ }^{2}, \quad I_{8}=\operatorname{tr} \boldsymbol{\Lambda}_{a}{ }^{2}, \quad I_{9}=\operatorname{tr} \boldsymbol{\Lambda}_{s}{ }^{3}, I_{10}=\operatorname{tr} \mathbf{C} \boldsymbol{\Lambda}_{s}=\operatorname{tr} \mathbf{C} \boldsymbol{\Lambda}$,

$I_{11}=\operatorname{tr} \mathbf{C}^{2} \boldsymbol{\Lambda}_{s}=\operatorname{tr} \mathbf{C}^{2} \boldsymbol{\Lambda}, \quad I_{12}=\operatorname{tr} \mathbf{C} \boldsymbol{\Lambda}_{s}^{2}, \quad I_{13}=\operatorname{tr} \mathbf{C}^{2} \boldsymbol{\Lambda}_{s}^{2}, \quad I_{14}=\operatorname{tr} \mathbf{C} \boldsymbol{\Lambda}_{a}^{2}, \quad I_{15}=\operatorname{tr} \mathbf{C}^{2} \boldsymbol{\Lambda}_{a}^{2}$,

$I_{16}=\operatorname{tr} \mathbf{C}^{2} \boldsymbol{\Lambda}_{a}^{2} \mathbf{C} \boldsymbol{\Lambda}_{a}, \quad I_{17}=\operatorname{tr} \boldsymbol{\Lambda}_{s} \boldsymbol{\Lambda}_{a}^{2}, \quad I_{18}=\operatorname{tr} \boldsymbol{\Lambda}_{s}^{2} \boldsymbol{\Lambda}_{a}^{2}, \quad I_{19}=\operatorname{tr} \boldsymbol{\Lambda}_{s}^{2} \boldsymbol{\Lambda}_{a}^{2} \boldsymbol{\Lambda}_{s} \boldsymbol{\Lambda}_{a}, \quad I_{20}=\mathbf{A} \boldsymbol{\Lambda}_{s} \mathbf{A}$

$I_{21}=\mathbf{A} \boldsymbol{\Lambda}_{s}^{2} \mathbf{A}, \quad I_{22}=\mathbf{A} \boldsymbol{\Lambda}_{a}^{2} \mathbf{A}, \quad I_{23}=\mathbf{A} \mathbf{C} \boldsymbol{\Lambda}_{s} \mathbf{A}, \quad I_{24}=\mathbf{A} \mathbf{C} \boldsymbol{\Lambda}_{a} \mathbf{A}, I_{25}=\mathbf{A} \mathbf{C}^{2} \boldsymbol{\Lambda}_{a} \mathbf{A}$,

$I_{26}=\mathbf{A} \Lambda_{a} \mathbf{C} \Lambda_{a}^{2} \mathbf{A}, \quad I_{27}=\mathbf{A} \boldsymbol{\Lambda}_{s} \Lambda_{a} \mathbf{A}, \quad I_{28}=\mathbf{A} \Lambda_{s}^{2} \Lambda_{a} \mathbf{A}, \quad I_{29}=\mathbf{A} \Lambda_{a} \Lambda_{s} \Lambda_{a}^{2} \mathbf{A}$,

$I_{30}=\operatorname{tr} \mathbf{C} \boldsymbol{\Lambda}_{s} \boldsymbol{\Lambda}_{a}, \quad I_{31}=\operatorname{tr} \mathbf{C}^{2} \boldsymbol{\Lambda}_{s} \boldsymbol{\Lambda}_{a}, \quad I_{32}=\operatorname{tr} \mathbf{C} \boldsymbol{\Lambda}_{s}^{2} \boldsymbol{\Lambda}_{a}, I_{33}=\operatorname{tr} \mathbf{C} \boldsymbol{\Lambda}_{a}^{2} \boldsymbol{\Lambda}_{s} \boldsymbol{\Lambda}_{a}$,

where the appearing symmetric and antisymmetric parts of $\boldsymbol{\Lambda}$ are defined in (2.7).

\section{Appendix B: Positive semi-definiteness of (2.16) and its consequences}

In the case of straight fibres direced in parallel to the $x_{1}$-axis, where $\boldsymbol{a}=(1,0,0)^{\mathrm{T}},(2.16)$ can be rearranged into the following form [6]:

$$
\begin{aligned}
& W_{(2)}^{\kappa}=\left(e_{11,1}, e_{22,1}, e_{33,1}\right) {\left[\begin{array}{ccc}
D_{11} & D_{12} & D_{12} \\
D_{12} & D_{22} & D_{23} \\
D_{12} & D_{23} & D_{22}
\end{array}\right]\left(\begin{array}{l}
e_{11,1} \\
e_{22,1} \\
e_{33,1}
\end{array}\right)+2 \beta_{3} e_{23,1}^{2}-2 \beta_{5} \Omega_{1,1}^{2}+} \\
&\left(e_{31,1}, \Omega_{2,1}\right)\left[\begin{array}{cc}
D_{66} & -D_{67} \\
-D_{67} & D_{77}
\end{array}\right]\left(\begin{array}{l}
e_{31,1} \\
\Omega_{2,1}
\end{array}\right)+\left(e_{12,1}, \Omega_{3,1}\right)\left[\begin{array}{cc}
D_{66} & -D_{67} \\
-D_{67} & D_{77}
\end{array}\right]\left(\begin{array}{l}
e_{12,1} \\
\Omega_{3,1}
\end{array}\right),
\end{aligned}
$$

where the appearing non-zero components of $\boldsymbol{D}$ are given as follows:

$$
\begin{array}{ll}
D_{11}=\beta_{1}+\beta_{2}+\beta_{3}+\beta_{4}+\hat{\beta}_{3}, \quad D_{12}=D_{13}=\beta_{1}+\beta_{2} / 2, \quad D_{23}=\beta_{1}, \\
D_{22}=D_{33}=\beta_{1}+\beta_{3}, & D_{44}=2 \beta_{3}, \quad D_{55}=-2 \beta_{5}, \quad D_{66}=D_{88}=2 \beta_{3}+\beta_{4}, \\
D_{67}=D_{89}=-\beta_{7} / 2, & D_{77}=D_{99}=-\left(2 \beta_{5}+\beta_{6}\right),
\end{array}
$$

and the components of the spin vector, $\Omega$, are defined in (2.19).

In view of the required positive definiteness of both the total stored energy, $W$, and its strain energy counterpart, $W^{e}$, one requires from $W_{(2)}^{\kappa}$ to be at least positive semi-definite $\left(W_{(2)}^{\kappa} \geq 0\right)$. By virtue of this requirement, Reference [6] concluded that the values of the moduli that appear in (2.16) should necessarily satisfy the following inequalities:

$$
\begin{aligned}
& \beta_{1} \geq 0, \quad \beta_{2}+\hat{\beta}_{3} \geq 0, \quad \beta_{3} \geq 0, \quad \beta_{4} \geq 0, \quad \beta_{5} \leq 0, \\
& \beta_{6} \leq-2 \beta_{5}, \quad \beta_{7}^{2} \leq-4\left(2 \beta_{5}+\beta_{6}\right)\left(2 \beta_{3}+\beta_{4}\right), \\
& \beta_{1}+\beta_{2}+\beta_{3}+\beta_{4}+\hat{\beta}_{3} \geq \frac{\left(\beta_{1}+\beta_{2} / 2\right)^{2}}{\beta_{1}+\beta_{3} / 2} .
\end{aligned}
$$
follows:

The procedure detailed in Section 6 of [14] observed that (B.1) can be described as 
$W_{(2)}^{\kappa}=W^{\Omega}+W^{E}$,

where the parts

$W^{\Omega}=D_{55} \Omega_{1,1}^{2}+D_{77}\left(\Omega_{2,1}^{2}+\Omega_{3,1}^{2}\right)$,

and

$W^{E}=\left(e_{11,1}, e_{22,1}, e_{33,1}\right)\left[\begin{array}{ccc}D_{11} & D_{12} & D_{12} \\ D_{12} & D_{22} & D_{23} \\ D_{12} & D_{23} & D_{22}\end{array}\right]\left(\begin{array}{l}e_{11,1} \\ e_{22,1} \\ e_{33,1}\end{array}\right)+D_{44} e_{23,1}^{2}+D_{66}\left(e_{31,1}^{2}+e_{12,1}^{2}\right)$,

of the internal energy depend on the directional derivatives of the spin and the strain components, respectively, along the fibre direction.

Being dependent on spin gradients only, $W^{\Omega}$ is expressed in terms of kinematic variables that were employed explicitly in the development of the polar linear elasticity due to Mindlin and Tiersten [16]. However, $W^{E}$ is expressed in terms of the additional kinematic variables, $e_{i j, 1}$, which are met neither in the theory presented in [16] nor in its generalisation attempted in Section 3 of [14]. It is accordingly noted that $W^{E}$ is the sum of three terms that contribute to deformation modes that resemble fibre-splay, fibre-twist and fibre-bending, respectively. On the other hand, the first term of $W^{\Omega}$ contributes to deformation that resembles fibre-twist while the second term to deformations resembling the corresponding fibre-bending mode.

Due to the presence of $W^{E}$, Reference [14] thus concluded that the unrestricted version of the present theory is generally incompatible with the theoretical framework of the Mindlin and Tiersten model [16], and that considerable and careful further investigation is required regarding the role that the observed, newly introduced kinematic variables, $e_{i j, 1}$, play in the unrestricted theory of polar linear elasticity of fibre-reinforced materials.

\section{Appendix C: Example of degenerated principal stretches}

Consider for simplicity a special case where (5.6) return a single degenerated principle stretch value, namely

$\lambda_{1}=\lambda_{2}=\lambda_{3}=\lambda$,

and, hence,

$I_{1}=3 \lambda^{2}=\sqrt{3 I_{2}}=3 \sqrt[3]{I_{3}}=3 I_{4}=3 \sqrt{I_{5}}$.

In that case, equation (5.7) are evidently not invertible, and the eigenvectors of $\boldsymbol{C}$ implied in (5.1) can be chosen arbitrarily.

Let us choose the eigenvectors of $\boldsymbol{C}$ to be identical to those of $\boldsymbol{\Lambda}_{s}$, so that

$\boldsymbol{\Lambda}_{s}=\sum_{i=1}^{3} t_{i} \boldsymbol{u}_{i} \otimes \boldsymbol{u}_{i}$,

and, for simplicity, let us assume that the eigenvalues, $t_{i}$, of $\boldsymbol{\Lambda}_{s}$ are all distinct. Then, it can be shown in analogy with (5.6), that 


$$
\begin{aligned}
& t_{j}=\frac{1}{3}\left\{K_{1}+2 \sqrt{K_{1}^{2}-3 K_{2}} \cos \frac{1}{3}[\theta+2 \pi(j-1)]\right\}^{1 / 2}, \\
& \theta=\arccos \left[\frac{2 K_{1}^{3}-9_{1} K_{1} K_{2}+27 J_{3}}{2\left(K_{1}^{2}-3 K_{2}\right)^{3 / 2}}\right], \quad K_{1}^{2}-3 K_{2} \neq 0,
\end{aligned}
$$

or

$t_{j}=\frac{1}{3} K_{1}+\frac{1}{3}\left(27 K_{3}-K_{1}^{3}\right)^{1 / 3}\left[\cos \left(\frac{2}{3} \pi j\right)+\sqrt{-1} \sin \left(\frac{2}{3} \pi j\right)\right], \quad J_{1}^{2}-3 J_{2} \neq 0$,

where

$$
K_{1}=I_{6}, \quad K_{2}=\frac{I_{6}^{2}-I_{7}}{2}, \quad K_{3}=\frac{I_{9}-I_{6}\left(I_{7}-K_{2}\right)}{2} .
$$

In the chosen simplicity route, consider only the case that (C.4) return distinct eigenvalues for $\boldsymbol{\Lambda}_{s}$, and note that, in that case, the set of algebraic equations (5.7) is replaced by the following:

$$
\begin{aligned}
& I_{20}=\boldsymbol{A} \boldsymbol{\Lambda}_{s} \boldsymbol{A}=t_{1} A_{1}^{2}+t_{2} A_{2}^{2}+t_{3} A_{3}^{2}, \\
& I_{5}=\boldsymbol{A} \boldsymbol{\Lambda}_{s}^{2} \boldsymbol{A}=t_{1}^{2} A_{1}^{2}+t_{2}^{2} A_{2}^{2}+t_{3}^{2} A_{3}^{2}, \\
& A_{1}^{2}+A_{2}^{2}+A_{3}^{2}=1 .
\end{aligned}
$$

Hence, solution of this algebraic system for $A_{i}^{2}$ and use (C.4) reveals that

$$
A_{i}=A_{i}\left(I_{6}, I_{7}, I_{9}, I_{20}, I_{21}\right) \text {. }
$$

In the same context, the set of equation (5.13) is replaced as follows:

$$
\begin{aligned}
& I_{8}=\operatorname{tr} \Lambda_{a}^{2}=2\left(r_{12}^{2}+r_{13}^{2}+r_{23}^{2}\right), \\
& I_{17}=\operatorname{tr} \Lambda_{s} \Lambda_{a}^{2}=t_{1}\left(r_{12}^{2}+r_{13}^{2}\right)+t_{2}\left(r_{21}^{2}+r_{23}^{2}\right)+t_{3}\left(r_{31}^{2}+r_{32}^{2}\right), \\
& I_{18}=\operatorname{tr} \Lambda_{s}^{2} \Lambda_{a}^{2}=t_{1}^{2}\left(r_{12}^{2}+r_{13}^{2}\right)+t_{2}^{2}\left(r_{21}^{2}+r_{23}^{2}\right)+t_{3}^{2}\left(r_{31}^{2}+r_{32}^{2}\right),
\end{aligned}
$$

and its solution for $r_{i j}^{2}=r_{j i}^{2}$ will provide

$$
r_{i j}=r_{i j}\left(I_{6}, I_{7}, I_{8}, I_{9}, I_{17}, I_{18}\right)=-r_{j i}, \quad(i \neq j) .
$$

It thus becomes clear that the remaining, unused classical invariants listed in (A.1) are redundant. This is because each of them can be expressed in terms of the nine spectral invariants $\lambda, t_{i}, r_{i j}, A_{1}$ and $A_{2}$, and, by virtue (C.2), (C.4), (C.5), (C.7) and (C.9), in terms of the nine independent invariants $I_{1}, I_{6}, I_{7}, I_{8}, I_{9}, I_{17}, I_{18}, I_{20}, I_{21}$. The later thus form a minimal set of independent invariants in this special case.

A number of additional similar examples may be formed by combining different cases of degenerated $\boldsymbol{C}$ - and/or $\boldsymbol{\Lambda}_{s}$-eigenvalues, and each of them can be handled by some similar, though different manner.

\section{References}

1. Adkins, J.E. and Rivlin, R.S., Large elastic deformations of isotropic materials X. Reinforcement by inextensible cords. Philos. Trans. R. Soc. London A 248, 201-223, 1955. 
2. Spencer, A.J.M. and Soldatos, K.P., Finite deformations of fibre-reinforced elastic solids with fibre bending stiffness. Int. J. Non-lin. Mech. 42, 355-368, 2007.

3. Spencer, A.J.M., Ronald Rivlin and invariant theory. Int. J. Eng. Sci. 47, 1066-1075, 2009. In "Mechanics, Mathematics and Materials" a Special issue in memory of A.J.M. Spencer FRS, Int. J. Eng. Sci. 47, 1055-1471, 2009, Selvadurai, A.P.S., Soldatos, K.P. and Rajagopal, K., eds.

4. Cosserat, E. and Cosserat, F., Théorie des Corps Deformables. Hermann, Paris, 1909.

5. Zheng, Q.S., Theory of representations for tensor functions: A unified invariant approach to constitutive equations. Appl. Mech. Rev. 47, 545-587, 1994.

6. Soldatos, K.P., Foundation of polar linear elasticity for fibre-reinforced materials. J. Elast. 114, 155$178,2014$.

7. Soldatos, K.P., Second-gradient plane deformations of ideal fibre-reinforced materials: implications of hyper-elasticity theory. J. Eng Math. 68, 99-127, 2010.

8. Dagher, M.A. and Soldatos, K.P., On small azimuthal shear deformation of fibre-reinforced cylindrical tubes. J. Mech. Mater. Struct. 6, 141-168, 2011.

9. Dagher, M.A. and Soldatos, K.P., Pure azimuthal shear deformation of an incompressible tube reinforced by radial fibres resistant in bending IMA J. Appl. Math. 79, 848-868, 2014.

10.Dagher, MA and Soldatos, K.P. Area-preserving azimuthal shear deformation of an incompressible tube reinforced by radial fibres. J. Eng. Math. 95, 101-119, 2015.

11.Farhat, A.F. and Soldatos, K.P., Cylindrical bending and vibration of polar material laminates. Mech. Adv. Mater. Struct. 22, 885-896, 2015.

12.Soldatos, K.P. and Farhat, A.F., On Reissner's displacement field in modelling thin elastic plates with embedded fibres resistant in bending. IMA J. Appl. Math. 81, 1076-1095, 2016.

13.Soldatos, K.P., On the characterisation of fibrous composites when fibres resist bending. Int. J. Solids Struct. 146-149, 2018.

14.Soldatos, K.P., On the characterisation of fibrous composites when fibres resist bending - Part II: Connection with anisotropic polar linear elasticity. Int. J. Solids Struct. 152-153, 1-11, 2018.

15.Soldatos, K.P., Aydogdu, M. and Gul, U., Plane strain polar elasticity of fibre reinforced functionally graded materials and structures. J. Mech. Mater. Struct. 14, 497-535, 2019.

16. Mindlin, R.D. and Tiersten, H.F., Effects of couple-stresses in linear elasticity, Arch. Ration. Mech. Anal. 11, 415-448, 1963.

17.Ting, T.C.T., Anisotropic Elasticity. Oxford University Press, New York, 1996.

18. Jones, R.M., Mechanics of Composite Materials, Taylor \& Francis, Washington, 1998.

19.Merodio, J., and Ogden, R.W., Material instabilities in fiber-reinforced non-linearly elastic solids under plane deformation. Arch. Mech. 54, 525-552, 2002.

20.Merodio, J. and Ogden, R.W., Instabilities and loss of ellipticity in fiber-reinforced compressible non-linearly elastic solids, Int. J. Solids Struct. 40, 4707-4727, 2003.

21.Merodio, J, and Ogden, R.W., A note on strong ellipticity for transversely isotropic linearly elastic solids. Q. J. Mech. Appl. Math. 56, 589-591, 2003.

22.Shariff, M.H.B.M., Physical invariant strain energy function for passive myocardium. Biomech. Model Mechanobiol., 12, 215-223, 2013.

23.Shariff, M.H.B.M., On the spectral constitutive modelling of transversely isotropic soft tissue: Physical invariants, Int. J. Engng. Science, 120, 199-219, 2017.

24.Shariff, M.H.B.M., Bustamante, R. and Merodio, J., A nonlinear constitutive model for a two preferred direction electro-elastic body with residual stresses, Int. J. Non-Lin. Mech., 2020119 , https://doi.org/10.1016/j.ijnonlinmec.2019.103352.

25.Shariff, M.H.B.M. and Merodio, J. Residually stressed two fibre solids: A spectral approach, Int. J. Engn. Sci. 148, 2020, 103205. 